\title{
3. USING PATHFINDER NETWORKS TO ANALYZE PROCEDURAL KNOWLEDGE IN INTERACTIONS WITH ADVANCED TECHNOLOGY
}

\author{
Douglas J. Gillan and Nancy J. Cooke
}

\begin{abstract}
Research on human cognition in complex tasks, such as interacting with advanced technology, requires the development and validation of new methods. This paper describes PRONET, a method for summarizing, representing, and analyzing event sequences. The first section outlines how the PRONET method can be applied to any sequence of events, with lessons learned from previous applications of the method. The second section presents demonstrations of the application of PRONET. In the first demonstration - a computer-based simulation of operant training - the PRONET analysis and representation clearly shows the change in the behavior of the simulation produced by changes in reinforcement contingencies, but also shows interesting aspects of behavior that were not affected. In the second demonstration - involving transfer of word processing skill-network-related and performance measures showed the expected pattern of positive transfer. In addition, the network of the far transfer participant suggested that she used task knowledge to search for conditions that would permit the correct action. Two previously-published examples showed the usefulness of the PRONET method in characterizing
\end{abstract}

\footnotetext{
Advances in Human Performance and Cognitive Engineering Research, Volume 1, pages $125-161$.

Copyright $\odot 2001$ by Elsevier Science Ltd.

All rights of reproduction in any form reserved.

ISBN: 0-7623-0748-X
} 
a hybrid event sequence consisting of environmental conditions and behavioral actions and a sequence of events from a team.

\section{INTRODUCTION}

The cognitive revolution has reached an uneasy middle age. Cognitive psychology replaced behaviorism as the dominant area of experimental psychology in the late 1960s. But, like so many of the revolutions in the '60s, cognitive psychology has failed to live up to the sparkling promise of its early days. Although some cognitive psychologists have claimed that cognitive theory and research has uncovered the workings of the mind (e.g. Pinker, 1997), that same research and theory has led to very limited applications (for more on this critique, see Gillan \& Schvaneveldt, 1999). If psychologists really understood the workings of the mind, it seems likely that the applications in such areas as education and training, the design of interfaces to advanced technologies, and testing and measurement would be overwhelmingly successful. Although certain demonstration programs in applying cognitive principles in education have shown promise (for examples, see Mayer, 1999), it is hard to imagine even the most optimistic cognitive psychologist claiming overwhelming success in applications.

Cognitive processes reveal themselves through behavior. In laboratory-based research, cognitive scientists typically measure such simple features of behavior as response time, accuracy of the response, or the type of a response. However, as cognitive engineers examine complex behavior in fields of practice, these simple measures may not accurately reflect the behavior. In other words, to study cognition in the wild (e.g. Hutchins, 1996), we must first create and validate new methodological approaches. These new methods must represent the complex behavior in a way that accurately reflects the behavior, and that also makes the data comprehensible and useful for making inferences about the cognitive processes underlying the behavior.

Interactions between people and advanced technologies, such as computers, provide a domain that is rich in both situated cognition and data recording capabilities. For example, usability tests frequently record behavioral events like keystrokes, mouse clicks, and errors that occur as a user interacts with software. In addition, recording and analysis of complex behavior can be useful in a variety of other cognitive engineering applications, including cognitive task analysis, training program development, and the design of cognitive aids. Complex behavioral data during productive tasks can be captured with relative ease in the work context for that task, using either videotapes of a user's interaction and commercially available or custom-built software for coding 
user responses (e.g. Owen, Baecker \& Harrison, 1994) and/or data logging software that automatically records the events and times and stores them in a computer file (e.g. Hammontree, Weiler \& Hendrich, 1995). Usability tests often collect the data unobtrusively as the video camera and/or event logging software run in the background while the user performs his or her tasks. In addition, event logging software enables usability testers to perform evaluations either in a controlled laboratory setting or in the user's environment. Unfortunately, analysis of the event records derived either from videotapes of user interactions or log files typically involves only a transcription of selected key segments, thereby relegating the bulk of the data and the potential wealth in latent information to the "archives" where it will remain until "needed". As a consequence, data remain unexamined that: (1) cognitive researchers might examine to study how users think during complex. productive tasks, and (2) cognitive engineers might examine as part of their applied work (for example, usability engineers could use the data to improve user interfaces).

Why does this unfortunate loss of information occur? Cognitive engineers ignore the bulk of the event logs because of immense difficulties associated with analyzing the data. Approaching the analysis of a record of behavioral data raises a myriad of questions: What is the most meaningful level of detail at which to parse the sequence of events? How can the very large event stream be summarized? How similar or different are two event logs? What aspects of the data are most critical for interface design considerations? Such questions, coupled with the more typical problems that arise when dealing with very large and complex data sets, make any extensive analysis of event logs seem insurmountable.

Recently, researchers have begun to develop methods designed to answer some of the questions raised above. An emerging area known as ESDA (exploratory sequential data analysis) has fostered the development of techniques and tools to facilitate the analysis of data that arise from sequences of events (Sanderson \& Fisher, 1994). In particular, ESDA techniques focus on making complex sequential data understandable through summarizing and statistically treating the data (for examples, see Bakeman \& Gottman, 1997; Bakeman \& Quera, 1995; Gottman and Roy, 1990), as well as, in some cases, presenting that summary in a visual format (for examples, see Cooke et al., 1996; Rauterberg, 1993, 1996). The ideal approach to dealing with complex sequential data would appear to be one that provided: (1) a statistical analysis to simplify and regularize the data, as well as (2) a way to perform inferential tests, and (3) an easy to interpret visual representation of the behavior. PRONET (Cooke et al., 1996) summarizes sequential event data based on creation of a matrix of transitions between events and the application of the 
Pathfinder algorithm (Schvaneveldt, 1990) to the matrix. The output of the Pathfinder algorithm applied to the event transition matrix can be presented in a visual format - a network with events represented as nodes and event transitions represented as links between nodes (for examples of other networkproducing methods that could be applied to behavioral sequences of user interactions, see Guzdial, 1993; Reisig, 1992; and Millen, Schriefer, Lehder \& Dray, 1997). In addition, the Pathfinder algorithm reduces the data by eliminating certain links and the resulting network can be characterized or compared to other networks by means of inferential statistical tests.

Because the PRONET approach meets the criteria outlined above - statistical treatment to simplify and provide inferential tests and a network format for presenting the behavioral sequence data, PRONET appears to be a promising method for analyzing event log file data. Accordingly, this paper provides readers with a detailed description of the method behind PRONET so that anyone wishing to make use of PRONET can do so. In addition, we review several very different demonstrations that show the capabilities of PRONET. The first demonstration analyzes behaviors of a digital entity in a computerbased simulation used in training (Graham, Alloway \& Krames, 1994). A second detailed demonstration is a more usual case of humans using complex technology, specifically, a human transferring her skill in using a novel version (to her) of a well-learned word processing application. The other demonstrations that we review - all of which examine the behavior of humans' interactions with advanced technology - are covered in less detail because their detailed descriptions are available in previous publications. These varied demonstrations show an important feature of PRONET - the flexibility in which researchers, educators, or designers might apply it.

\section{PRONET: PROCEDURAL NETWORKS}

In this section we provide a detailed description of PRONET, a method for summarizing and representing sequential event data. The sequence of events may be human or animal behavior, machine events, or environmental events. PRONET represents the event sequence in terms of a network of nodes (the events) and links (based on the transitions between events) similar to the result of Markov analysis or a Petri net representation; however, the PRONET approach differs from Markov and Petri net representations in that the Pathfinder network scaling algorithm (Schvaneveldt, Durso \& Dearholt, 1989; Schvaneveldt, 1990) - the statistical basis for the PRONET method - provides a statistical method for selecting certain sequences of behaviors to maintain in the network and other sequences of behavior to eliminate (see Cooke \& Gillan, 
1999, for a review of these methods). Pathfinder analyzes a set of pairwise proximities as a node-link structure. Below, we outline the general procedure for PRONET (for more details, see Cooke et al., 1996; and Cooke \& Gillan, 1999).

\section{Collecting, Coding, and Organizing the Data}

The PRONET method can be conducted with any type of sequential data. In the context of an interaction between a human and technology, data will most likely take the form of user, system, and environmental events. The data ma also be collected by a variety of means, including videotaped behavior, thinkaloud verbal reports, eye movements, gestures, or event logging software.

Encoding the events, the most difficult and time-consuming aspect of PRONET, involves transforming the continuous stream of raw event data into a series of discrete categorized events. In order to enhance data summary, categories should be fewer in number than actual unique events. Decisions about event categories are important and highly dependent on the purpose of the data analysis. As is illustrated in some of the demonstrations described in this paper, more than one encoding scheme may make sense. Accordingly, an important factor in selecting a coding scheme is the question asked of the data. Cooke et al. (1996) proposed six heuristics to aid in developing the coding scheme:

- Minimize the number of event categories in order to reduce data and allow more observations per node.

- Preserve distinctions that are meaningful for the goals of analysis.

- Consider ignoring or merging into one category events that are uninteresting or less important than other events.

- Consider merging events into a single category if they always co-occur.

- If an event does not change across encoded protocols, then there is no need to represent that event as a node.

- Generate an event taxonomy and iterate on a subset of the data in order to decide on the appropriate level of abstraction.

Following encoding, PRONET requires the series of discrete categorized events be transformed into a co-occurrence or event transition matrix. In this matrix, the rows and columns represent events, and entries in the matrix represent the frequency with which the event in the column followed the event in the row. Although raw co-occurrence frequencies can be submitted to Pathfinder, they do not take into account differences in the absolute frequency with which single events occurred. For that reason, we recommend the use of conditional transition probabilities. 
A PRONET analyst will need to make several decisions about these transitions. PRONET also permits analysts to define events as a string of behaviors of any size and to examine transitions of events that follow one another with any size lag. The nature of the task or the characteristics of the users may provide good reasons to consider some of these higher-order transitions. In the event that transitions of several varying lags are used, they may be combined in a proximity matrix, but it may make sense to weight them differently. For instance, longer lags could be given less weight than shorter lags. In addition, the analyst should consider eliminating spurious transitions including those that occur between subtasks or that consist of an event that occurs independent of the preceding event.

\section{Applying Pathfinder to the Transition Matrix Data}

Following the creation of an event transition matrix, a PRONET analyst would next apply the Pathfinder algorithm to the data. The Pathfinder network scaling technique (Schvaneveldt, Durso \& Dearholt, 1989; Schvaneveldt, 1990) is a statistical method for structural modeling, much like cluster analysis (Johnson, 1967) and multidimensional scaling (Kruskal \& Wish, 1978). These structural modeling methods translate pairwise proximity estimates for a set of items (collected as relatedness ratings, co-occurrence of sorted items, or recall order) into either a spatial or graphical configuration. PRONET has two special characteristics: (1) the items are events, including behavior produced by a human, a nonhuman animal, or a non-living system such as a computer that can generate events, and (2) the proximities among events are based on transition frequencies or probabilities between event pairs.

The various structural modeling methods reveal the latent structure in the proximity data. The methods differ from one another in terms of the representational format and assumptions underlying the method of generating the representation. Pathfinder assumes that the proximity estimates reflect information about distance in a network structure.

Pathfinder links represent relations, and a weight associated with the link and derived from the original proximity data reflects the strength of the relation. In contrast, the nature or meaning of the relation is unidentified. Links can also be directed if proximity estimates are asymmetrical, as one typically finds in proximities derived from event transitions. Nodes can also be unconnected if the estimates between an item and all other items fail to exceed a minimum strength criterion set by the experimenter.

A completely connected network can be used to represent (without any data reduction) the original proximities. One of the benefits of the Pathfinder algorithm, however, is in its data reduction properties. The algorithm eliminates 
various links in order to reduce the data and facilitate comprehension of the resulting network. Basically, if a link exceeds the minimum strength criterion set by the experimenter, it is included by the algorithm if the minimum distance path (chain of one or more links) is greater than or equal to the distance indicated by the proximity estimate for that pair.

Two parameters, $r$ and $q$, determine how network distance is calculated and therefore, affect the ultimate density of the network. The parameter $r$ is based on the Minkowski $r$ metric (Dunn-Rankin, 1983) so that, when $r$ equals infinity, path distance is equivalent to the maximum weight along the path. The parameter $q$ specifies the maximum number of links allowed in a path. The KNOT software ${ }^{1}$ which implements the algorithm, sets default values for $r$ and $q$ of infinity and number of items -1 , respectively. The default values (i.e. $r=$ infinity; $q=$ number of items -1 ) result in networks of minimum complexity. Further, when $r=$ infinity, only ordinal assumptions of the proximity data are required. Decreasing the $q$ value results in networks of greater complexity, which may be undesirable when dealing with complex event data - the networks will be sufficiently complex with the default parameters. However, the value of $r$ may be set at 1 or 2 to reflect that probabilities as the proximity measure have ratio properties. When $r=1$ the path distance is computed by summing the link weights along a specific path; when $r=2$, the path length is computed as the Euclidean distance. Setting $r$ to either value will likely result in a more complex network, but may seem warranted from a measurement perspective.

When the data analyzed using Pathfinder are event transition frequencies or probabilities, they can be treated as similarities by the algorithm with high frequencies corresponding to high similarity. It is also necessary to identify the data as asymmetrical. Finally, the analyst has the opportunity to specify a minimum cutoff for frequencies or probabilities. Transitions with frequencies or probabilities below this cutoff will not be represented as links in the network. This step actually eliminates quite a few of the transitions that would otherwise be linked. Other links will later be eliminated by the distance rule associated with the Pathfinder algorithm and described above. As the complexity of the network (i.e. number of links) increases, the number of indirect paths between two nodes increases, leading the Pathfinder algorithm to eliminate direct paths that are "less efficient", thereby resulting in greater relative data reduction.

The output of Pathfinder is a list of nodes and weighted links, as well as the network representation in which events are represented as nodes. Directed links indicate transitions that occur between nodes. Readers should keep in mind that a path of two or more links is not an indication that that sequence of three or 
more events ever occurred contiguously. On the other hand, each consecutive pair of linked events has occurred together at least once.

Nodes may fail to be connected for three reasons. If nodes A and B are not linked in a PRONET network, (1) event A may not have ever been followed by event B in the observed sequence, (2) event B may have followed event A but at such a low probability that it was eliminated by the cutoff criterion, or (3) the probabilities in an indirect path between the two events (e.g. between $\mathrm{A}$ and $\mathrm{C}$ and $\mathrm{C}$ and $\mathrm{B}$ ) were enough higher than in the direct path between $\mathrm{A}$ and $\mathrm{B}$ that the direct path was eliminated.

Pathfinder has several features that make it well-suited to the analysis of sequential data found in behavioral records. First, it is more flexible than other scaling techniques in that the output of Pathfinder is not constrained to hierarchical configurations as is the case for most clustering techniques. Second, Pathfinder can represent asymmetric relations which are characteristic of sequential event data. Finally, various empirical results have supported the psychological validity of Pathfinder representations (Cooke, Durso \& Schvaneveldt, 1986; Cooke, 1992).

\section{Interpreting the PRONET Network}

Once a completed network is in hand, an analyst can make use of several procedures, both qualitative and quantitative, for interpreting the results. A qualitative interpretation entails examining each network in terms of nodes, links, and overall structure. Nodes that are disconnected from the rest of the network indicate omitted or infrequent events. This information is often very informative in the design of advanced technology (e.g. underused menu options or commands in a computer interface).

Links between nodes provide an indication of procedures that the user undertakes in accomplishing tasks. When events that are environmental or system-initiated are included, if-then or production rules can be derived from cases in which a system-event and user-event are strongly linked. In addition, chains of interconnected events and cycles may also be worthy of further examination. Finally, highly central nodes that are connected to many other nodes provide an indication of centrality or importance of events in the procedure. For example, in developing a computer-based training system, a highly central event involving access to help would suggest that the trainee found the system difficult to use. In examining the overall structure of the network, analysts should look for subnetworks (a set of events with multiple within-set links). Distinct subnetworks that have few connections to the rest of the network indicate events that occur together, as in a subsystem, but that do not typically occur with events outside of the subsystem. 
A PRONET analyst might use the following heuristics to aid in the interpretation of the networks:

- Network nodes correspond to events that occurred during a session.

- Each node is associated with a frequency (the number of times it was executed in the session or series of sessions). Frequencies can be categorized and coded for graphical presentation. Node frequency provides an indication of events that never occurred or occurred a great deal. This information is very useful for determining software functions never used or used often.

- Links between nodes in the network have a spatial direction which corresponds to the temporal ordering of the events during the session. This means that links can go in one or both directions. The direction indicates the order in which the two events (nodes) occurred in the interaction.

- Links are also associated with a weight indicating the strength of the link. Strong links are those for which the sequence occurred frequently or with high conditional probability.

- Sequences that occur frequently provide information about the user's typical strategies for the task.

- Networks can be examined for events that are linked to specific other events of interest to identify the events that precipitated a problem.

- Networks can be examined to identify events that are highly central (i.e. linked to several other events). This can indicate the importance of that event.

- The software will also provide quantitative indices representing the similarity of two or more networks (shared nodes, links, etc.).

At a quantitative level, the output of Pathfinder can be analyzed in a number of ways. Node frequency is not represented in the output, but is available in the transition matrix. It is possible to graphically represent this value, however, by making frequent nodes larger or darker. Other values that are available include number of links and weights of links. These values can be examined within a single network or they can be compared across networks. The KNOT program also contains a similarity measure, based on the proportion of shared links in two networks ranging from 0 (complete dissimilarity) to 1 (identity), which provides a quantitative value describing the similarity between two network structures (Goldsmith \& Davenport, 1990). This measure can be used to compare procedural networks of behavior associated with two different interfaces, two different training programs, or two different experience levels. Behavior can also be assessed by comparing an individual network to a referent structure which may represent some ideal behavior or the behavior of an experienced user. 


\section{PRONET DEMONSTRATIONS}

\section{Demonstration I: Computer-Based Simulation of Operant Training}

The first demonstration examines an aspect of advanced technology - a computer-based simulation of operant conditioning called Sniffy the Rat (Graham, Alloway \& Krames, 1994). For this demonstration, we used PRONET to summarize the simulation itself by examining Sniffy's behavior at five phases during typical operant training of a bar press response. The value of analyzing this simulation includes: (1) the limited set of behaviors in the simulated operant training situation provides a straightforward demonstration of PRONET; (2) the changes in behavior during operant training were programmed into the simulation; as a consequence, they are predictable and reliable, so that if PRONET is a valid analytical tool, the resulting networks should reflect those changes; and (3) the demonstration illustrates the use of PRONET to summarize sequences of computer-generated events, and as a method for validating computer-based simulations.

\section{Method}

The method used for this demonstration of PRONET mirrored the description above. We observed and recorded seven behaviors produced by Sniffy, the computer-based simulation of a rat, during five sessions. The Sniffy simulation shows a rat in an operant conditioning chamber (i.e. Skinner box) that has a bar for pressing, a light, a water spout, and a food hopper which appear to be at the far end of the chamber. The seven behaviors were: (1) sniffing the environment, (2) scratching itself, (3) standing in any place in the simulated operant conditioning chamber other than when standing was part of a bar press response, (4) licking itself, (5) drinking water from the water spout shown as part of the simulation, (6) pressing the bar shown, and (7) eating food from the hopper. This was the entire behavioral repertoire of Sniffy. The limited number of unique behaviors made the use of Sniffy well suited for the present demonstration. However, we do not claim that the results of our demonstration are generalizable to real rats receiving real operant training.

The five sessions represent 20 minute segments of different phases of typical operant training. Table 1 summarizes the five sessions. The first session involved observing Sniffy in an untrained state for 20 minutes. The simulation was set up so that Sniffy had received no prior training. Pressing on the bar (either by Sniffy or by the user moving the cursor over the bar and clicking on the mouse button) resulted in delivery of a food pellet (in the simulated world). However, Sniffy had not been given any experience with this contingency. Prior 
Table 1. The Five Sessions Used in the Analysis of the Sniffy the Rat Computer-Based Simulation.

\begin{tabular}{|c|c|c|c|c|c|}
\hline & Session 1 & Session 2 & Session 3 & Session 4 & Session 5 \\
\hline $\begin{array}{l}\text { Type of } \\
\text { Training }\end{array}$ & Untrained & CRL & VR-10 & $\begin{array}{l}\text { Early } \\
\text { Extinction }\end{array}$ & $\begin{array}{l}\text { Late } \\
\text { Extinction }\end{array}$ \\
\hline $\begin{array}{l}\text { Training } \\
\text { Procedure }\end{array}$ & $\begin{array}{l}\text { No shaping of } \\
\text { bar press } \\
\text { received. } \\
\text { Food given } \\
\text { after every } \\
\text { bar press. }\end{array}$ & $\begin{array}{l}\text { Shaping of } \\
\text { bar press } \\
\text { previously } \\
\text { given. Food } \\
\text { given after } \\
\text { every bar } \\
\text { press. }\end{array}$ & $\begin{array}{l}\text { Food given } \\
\text { after every } \\
10 \text { th bar } \\
\text { press, on } \\
\text { average. }\end{array}$ & $\begin{array}{l}\text { No food after } \\
\text { bar presses. }\end{array}$ & $\begin{array}{l}\text { No food after } \\
\text { bar presses. }\end{array}$ \\
\hline
\end{tabular}

to the second session, we shaped Sniffy to press the bar using successive approximations of the desired response. Also prior to the second session, the Sniffy simulation was run for 30 minutes on a continuous reinforcement (CRF) schedule. Thus, the second session involved 20 minutes of observing and recording Sniffy's responses, following the shaping and 30 minutes of CRF experience.

Before the third session, the Sniffy simulation was gradually extended to a Variable Ratio 10 (VR10) reinforcement schedule (i.e. on average Sniffy would receive one pellet of food for every 10 bar presses). The training involved giving Sniffy experience with a Fixed Ratio 2 (FR2), FR3, FR5, FR7, FR9, and FR10, prior to giving her 50 minutes of experience with a VR10 schedule. During the third session, we observed and recorded Sniffy during the last 20 minutes of that VR10 training. During the two extinction sessions, Sniffy had the link between bar press and food delivery eliminated. In fact, no response by Sniffy or the experimenters could produce the delivery of food. In Session 4 (Early Extinction), we observed and recorded Sniffy's behaviors for the first 20 minutes of the Extinction procedure. Then, prior to Session 5 (Late Extinction), the simulation was run for 2 hours under conditions of extinction. Session 5 consisted of the next 20 minutes of extinction training. To create each session, we gave Sniffy the experience described, then saved the simulation as a unique file. Thus, this demonstration involved observing five different Sniffy files, one for each phase of Sniffy's training.

The sequences of the seven responses generated during the five phases of operant conditioning were used to create transition matrices and conditional 
probability matrices as described in the previous section. Then, the Pathfinder algorithm was applied to the conditional probability matrices to generate network solutions.

\section{Results}

Figure 1 shows the PRONET solutions for each of the five stages of operant training. In the graphic representations of each network, the frequency of each response is coded by the size of the network node (i.e. the box), the thickness of the lines making up the box, and a number in the box. The conditional probability of a transition from one response to the next served as the basis for the link weights which are coded by the thickness of the lines and the number
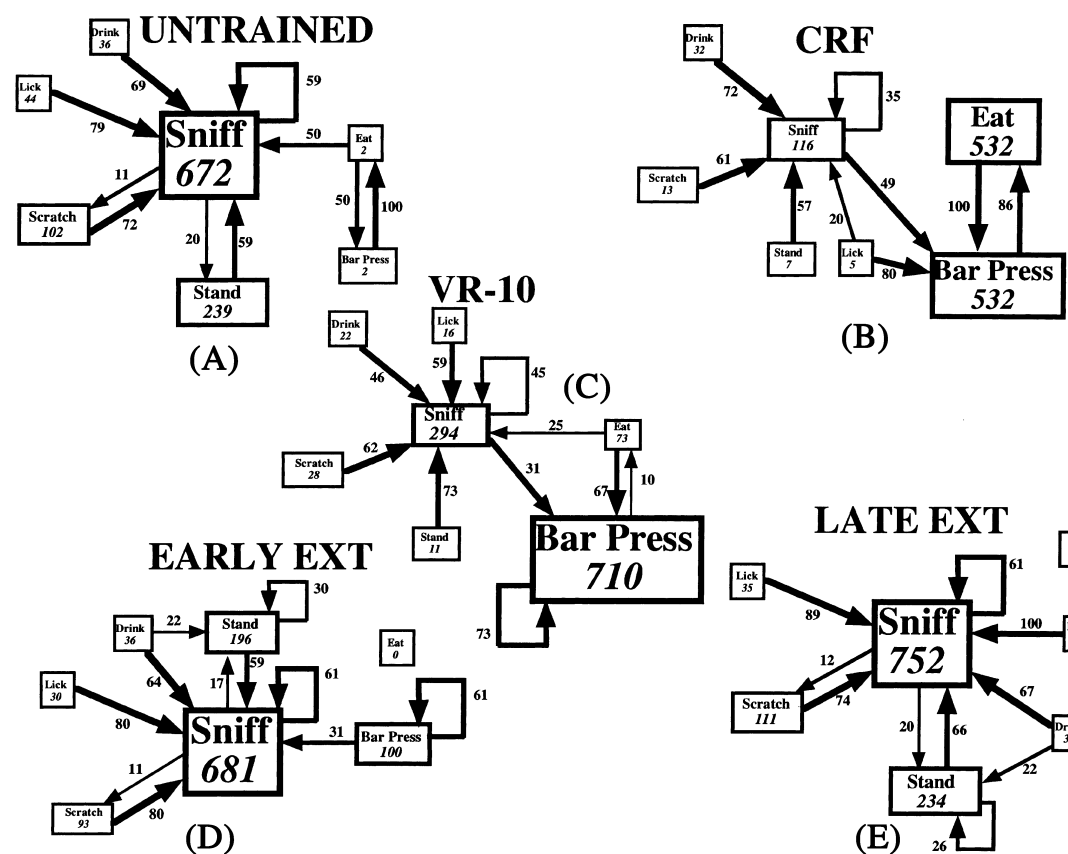

(B)

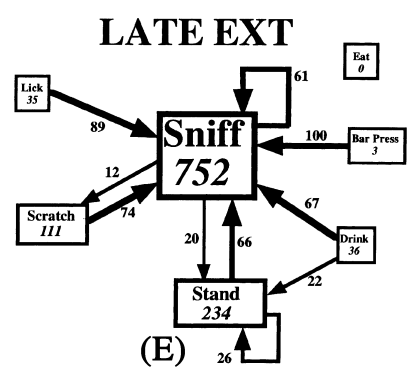

Fig. 1. Pathfinder networks based on the conditional transition matrices from the five session of operant training in Demonstration I - Pretraining, continuous reinforcement, variable ratio 10 , the first 20 minutes of extinction, and the final 20 minute of extinction. The frequency of each behavior is represented by the size of the nodes, the thickness of the lines for the box, and numbers within the box; the level of conditional probability is represented by the thickness of the lines linking nodes and the number next to the line (which is $100 \times$ the conditional probability). 
next to the line (which is 100 times the conditional probability). In each network, links with a weight less than 10 were eliminated.

Examining the PRONET network from the Pretraining session (Fig. 1A) shows that sniffing was the primary response prior to training, with bar pressing and eating being rare. All of the responses were very likely to be followed by sniffing except for bar pressing. Then, once delivery of food was made contingent on pressing the bar in the CRF session (Fig. 1B), the frequency of bar pressing and eating increased dramatically, with the bar pressing typically followed by eating which was always followed by pressing the bar. The frequencies of sniffing, standing, and scratching all decreased, whereas licking and drinking did not decrease as precipitously. Sniffing was still more likely response to follow all of the other behaviors than eating or bar pressing. In the VR10 session (Fig. 1C), bar pressing became the dominant response, and pressing the bar was very likely to be followed by more bar pressing. As would be expected from a VR10 schedule of reinforcement, bar pressing was followed by eating with a conditional probability of $10 \%$. Compared to the CRF session, the frequency of sniffing increased, but remained much lower than in Pretraining. Scratching, standing, licking, and drinking remained at low levels. As during the Pretraining and CRF sessions, behaviors other than bar pressing were likely to be followed by sniffing. Even eating had a fairly sizable conditional probability of being followed by sniffing during this session.

During the first 20 minutes of extinction (Fig. 1D), Sniffy greatly decreased bar pressing, with corresponding increases in sniffing, standing, and scratching. Drinking and licking remained at low levels. Because this was an extinction session, eating did not occur. As in the previous sessions, behaviors were likely to be followed by sniffing. The major exception was bar pressing - a bar press was most likely to be followed by another bar press, a remnant of the VR10 training. Later in extinction (Fig. 1E), bar pressing had returned to a very low frequency, with sniffing, standing, and scratching returning to their high levels observed in pretraining. As in all sessions, most responses were most likely to be followed by sniffing.

The PRONET networks of the five phases of Sniffy, the virtual rat's operant training show that the operant contingencies modified Sniffy's behavior - bar pressing and eating became the dominant responses during CRF training, with bar pressing gaining sole dominance during VR10 training. This pattern of change in behavior is typical of operant conditioning and is evident in the cumulative record that accompanies the simulation. Thus, the results of this demonstration support the validity of the PRONET method.

The PRONET analysis also reveals the changes in the frequency and likelihood of other behaviors as bar pressing increased. As bar pressing 
increased in both frequency and in the likelihood of being the response to follow eating, sniffing, or a previous bar press, the frequency of other types of behavior decreased, with the bulk of the decrease coming from sniffing, standing and scratching. The frequencies of licking and drinking did not change as much throughout the five sessions. Another aspect of the behavior in the simulation that was not very sensitive to the reinforcement contingencies was the likelihood of a transition to sniffing following any response. Most responses tended to be followed by sniffing (except for those directly involved in the reinforcement contingency, bar pressing and eating) in all five sessions. These findings show that PRONET can reveal the relations among the entire set of events in a sequence.

If Sniffy had been a live rat rather than a simulated one, sniffing around a chamber could be interpreted as an exploratory behavior. Thus, the propensity for a rat to sniff frequently in the Pretraining session and to return to sniffing after engaging in any other behavior would suggest that the rat had a general purpose procedure, "If behavior A is completed, then explore". A researcher observing a real rat whose behavior resulted in PRONET networks like the ones in the five sessions here might reasonably infer that this exploration procedure remained intact throughout the five sessions. To continue to have a strong exploration procedure despite the contingency between bar pressing and reinforcement and the consequent increase in bar pressing and decrease in the frequency of sniffing in the CRF and VR10 sessions would seem to be unexpected. That is to say, this aspect of the simulation appears to have low face validity. Thus, in addition to showing that the PRONET networks provide simplified and interpretable summaries of a sequence of behaviors, this analysis points out another valuable use of the PRONET method - to evaluate sequences of the behavior of computer-based simulations.

\section{Demonstration II: Transfer of Procedural Knowledge}

Users of technology often must transfer knowledge of how to use a system from one task environment to another. For example, a user who learns to use a word processing application in an office will try to use the same application for tasks at home. In today's world of multiple applications and computer platforms, users may also have to transfer between computer systems. For example, an experienced user of Microsoft Word for Windows might have to use WordPerfect or Word for the Macintosh. Can PRONET networks help researchers identify the procedures that underlie successful transfer, thereby giving guidance to designers so that they can develop interfaces that make it easier for users to transfer knowledge? 


\section{Method}

The participants in this study were students at New Mexico State University: one of whom had limited computer experience (hereafter referred to as the Baseline participant), one who had extensive experience with Microsoft Word for Windows and with Microsoft Windows 95 (hereafter referred to as the Windows-to-Windows Transfer participant), and one who had extensive experience with Microsoft Word for the Macintosh, but had no prior experience with Word for Windows and little experience with Microsoft Windows (hereafter referred to as the Mac-to-Windows Transfer participant). The research task required the participants to use Windows95 and Word for Windows 7.0 to type a business letter. A scenario was given to them which specified the requirements of the letter, including specific margins and font type, creation of two tables, pagination requirements, and the purpose of the letter and basic contents. In addition, we asked the participants to save the letter, print it, create a new folder, and move the letter to the new folder. We recorded the users' actions both by videotaping and by use of a speciallydesigned program for logging Windows events. During the task time (approximately 35 minutes) the test coordinator observed the participant via the TV monitor in a room adjacent to the testing room. Once a participant had completed the task, the test coordinator replayed sections of the video for that participant and asked him or her to provide a retrospective verbal report. In general, the test coordinator focused on aspects of the task that seemed problematic for the participant and areas in which the participant's goals or intentions had to be clarified. These data were collected largely to supplement the $\log$ file and video data. The retrospective report required approximately 15 minutes.

The behavioral sequence data were treated as described in the section that outlines the PRONET procedure above. Here we will detail the unique steps in data treatment for this research. We first coded each event for each participant based on the output from the event logging software, the associated videotape of a participant's interaction, and, when necessary, the retrospective verbal protocol. Because certain issues concerned with user cognition and with usability may focus more on the user's purpose or goals (i.e. the functional level), whereas other issues may center more on the actions that the user performed (i.e. the operational level), we decided to employ coding schemes to correspond to each of these levels. These levels of abstraction mirror the keystroke and functional level models used in GOMS analysis (Card, Moran \& Newell, 1983). Because of the relative ease of interpretability, the data from the high level coding are presented in this report. Next, we transformed each coded event sequence into a frequency matrix in which events were represented along 
the rows and columns, then into conditional probability matrices. Finally, these conditional transition probabilities were analyzed using the Pathfinder algorithm with the default parameters of $q=n-1$, and $r=\infty$. (In these analyses we did not consider transitions that occurred less than $10 \%$ of the time.)

In addition to analyzing the data from the participants, we developed a task analysis of the letter writing task. The task analysis was used to generate an optimized sequence of behaviors which was then analyzed using PRONET. The result was an optimized network of behaviors for the task that served as a comparison for the networks from the human participants.

\section{Results}

In addition to the PRONET analysis, we measured two aspects of performance: the time to complete the task in minutes, and the quality of the final product (assessed by the mean judgment of three blind judges on a scale of $1=$ poor to $10=$ good). The Windows-to-Windows participant was the fastest, taking 30 minutes to complete the task, with the Mac-to-Windows participant requiring 37 minutes and the Baseline participant taking 48 minutes. The quality of the participants' letters were rated as 7.3, 8.3, and 5.0, respectively. The better performance by the Mac-to-Windows participant than the Baseline participant indicates positive transfer in this task. In fact, the quality of the letter produced was slightly better for the Mac-to-Windows participant than even the Windowsto-Windows, further supporting the idea of positive transfer from Microsoft Word on the Macintosh to Word for Windows.

Another way to compare the three participant's behavior as they used Word for Windows to write the letter is to look at the similarity between each of their PRONET networks and the optimized network based on the task analysis. Table 2 compares the networks in terms of network similarity, based on the proportion of shared links and difference between the observed and expected number of links in common. Network similarity takes into account the total number of links in the networks whereas the difference between the observed and expected number of links in common does not. Also, the difference between observed and expected links or between the observed and expected similarity tends to be more sensitive than the raw link and similarity measures. The absolute values in Table 2 are low because (1) a relatively open-ended task with many possibilities for different actions produces a low proportion of shared events between any two individuals, resulting in low overall similarities between the individuals' networks, and (2) the measure used here was the amount by which the observed similarity exceeded the expected similarity. 
Table 2. Observed - Expected Network Similarity Measures for Each Participant Compared to the Optimized Performance Based on Task Analysis (Higher Values Indicate High Similarity.

\begin{tabular}{lcc}
\hline & $\begin{array}{c}\text { Observed-Expected } \\
\text { Network Similarity }\end{array}$ & $\begin{array}{c}\text { Observed-Expected } \\
\text { Links in Common }\end{array}$ \\
\hline Baseline & 0.01 & 0.36 \\
Windows-Windows & $0.10^{* *}$ & 4.19 \\
Mac-Windows & $0.03^{*}$ & 1.82
\end{tabular}

$* p<0.05$; ** $p<0.01)$ and Observed - expected links in common for each participant compared to the optimized performance (higher values indicate more links in common) in Demonstration II.

Having many paths to reach a given outcome typically produces differences across users in terms of which actions were executed and the frequency of the transitions between actions. As a consequence, the resulting networks differ a great deal in their node composition - actions that were never executed by a participant will not occur as a node in the network and, as a consequence that node cannot be linked to any other node. However, the data agree with the other performance measures in that they show higher similarity between the optimized performance and the two experienced users than between the optimized performance and the baseline user, with the highest similarity by the Windows-to-Windows participant.

Examining the specific structures of each network can be most useful for interpretation and for identifying specific procedural knowledge that a user may have (or gaps in procedural knowledge). Figures 2-4 shows the high-level functional networks for each user during the session in which they created a letter using Word for Windows. In the networks, nodes indicate events occurring in the participant's interaction with the computer and are coded by shape to indicate the event category (from the coding scheme) and by size, border thickness, and pattern to indicate the frequency of the event. Links indicate that one event followed another in a sequence in the order indicated by the arrows. The thickness and dashedness of the lines provides an indication of link strength which is based on the conditional probability of that particular transition.

Figure 2 shows the high-level network from the analysis of the Baseline user's behavior. The network consists of 14 nodes and 26 links that summarize 14 functions which occurred a total of 255 times with 34 unique transitions between functions. The PRONET analysis produced a simple network. The 
Transition Probabilities:
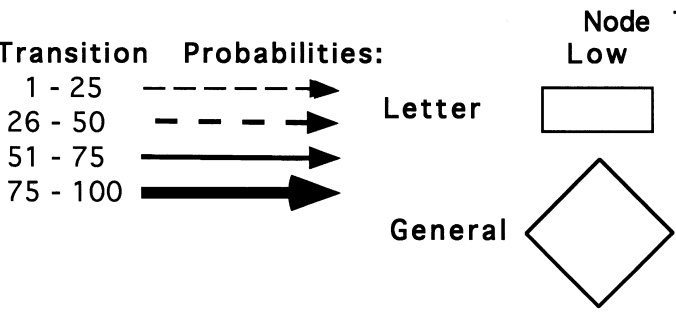

Types and Frequencies:

General

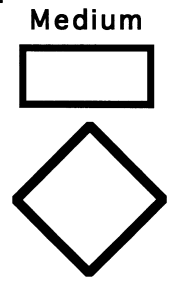
High

Table
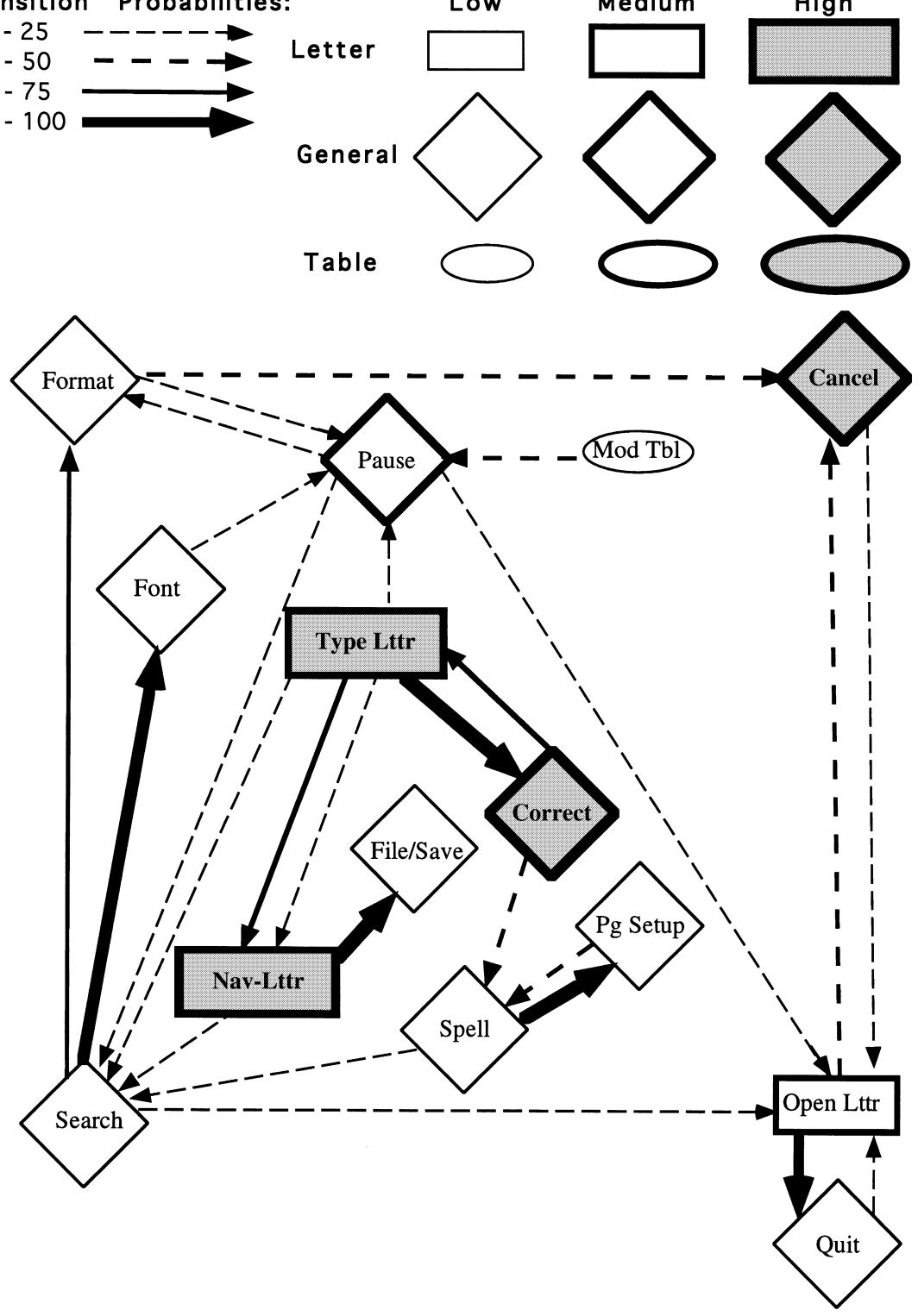

Fig. 2. Pathfinder network based on the conditional transition matrix for the Baseline participant in Demonstration II. The shading of the nodes represents the type of behavior; the size of the node indicates the frequency of the behavior; and the thickness and dashedness of the lines linking nodes represents the level of conditional transition probability. 
network indicates that this participant created the table without using the software's table-creating capabilities, nor did she use Help to find out how to access those capabilities. One explanation of this pattern of behavior would be that she did not know that Word has special purpose functions for creating tables. In addition, Correct, Pause, and Search are central to the Baseline user's network: (1) Type Lttr connects strongly with all three functions; and (2) Pause and Search have numerous links with other functions. The centrality of pausing and searching suggest that this participant frequently stopped to think about what to do next and to find information and, further, that these interruptions were not confined to one type of function, but were more universal. The pattern of the Baseline user's network - with much pausing, searching, and correcting, as well as the failure to use the relevant table-creating functions - are consistent with a low level of experience with computers and help explain her poor performance.

Figure 3 shows the high-level network, consisting of 22 nodes and 33 links, from the Windows-to-Windows user's transition probability data (representing the data from 22 functions which occurred a total of 183 times with 69 unique transitions). Several key features of the network stand out. First, the functions related to the letter tend to be interconnected and can be seen in the upper half of the large network. Several general functions are linked to the letter-related functions, but not the table-related functions - Close, Save, Page Setup, Page Break, Spell Check, Quit, Pause, and Search. Perhaps the most interesting of these are Pause and Search. This participant appears to have paused to compose the letter (the Pause -Type Lttr link), and in deciding whether to save or quit the file. Searching occurred as she typed and navigated through the letter. In addition, she searched in order to find how to open the table. The functions related to the table, found in the bottom half of the network, also tend to be interconnected and not directly connected to those for the letter. The general functions linked exclusively to table-related functions - including Select, Copy, Cut, and Paste - indicate that she reused (or attempted to reuse) parts of the table to a greater extent than she did parts of the main body of the letter. Finally, the general function, Correct, bridges between the letter- and table-related functions. Note however, that Correct is linked only to typing the letter among the letter-related functions, but is linked to typing, navigating, and modifying the table. The small highly interconnected subnetwork of Correct, Mod Tbl, Nav-Tbl, and Type Tbl suggests that this user made many corrections as she created the table. This network is consistent with a user knowledgeable about basic word processing, but not at creating tables as part of the word processing application. 


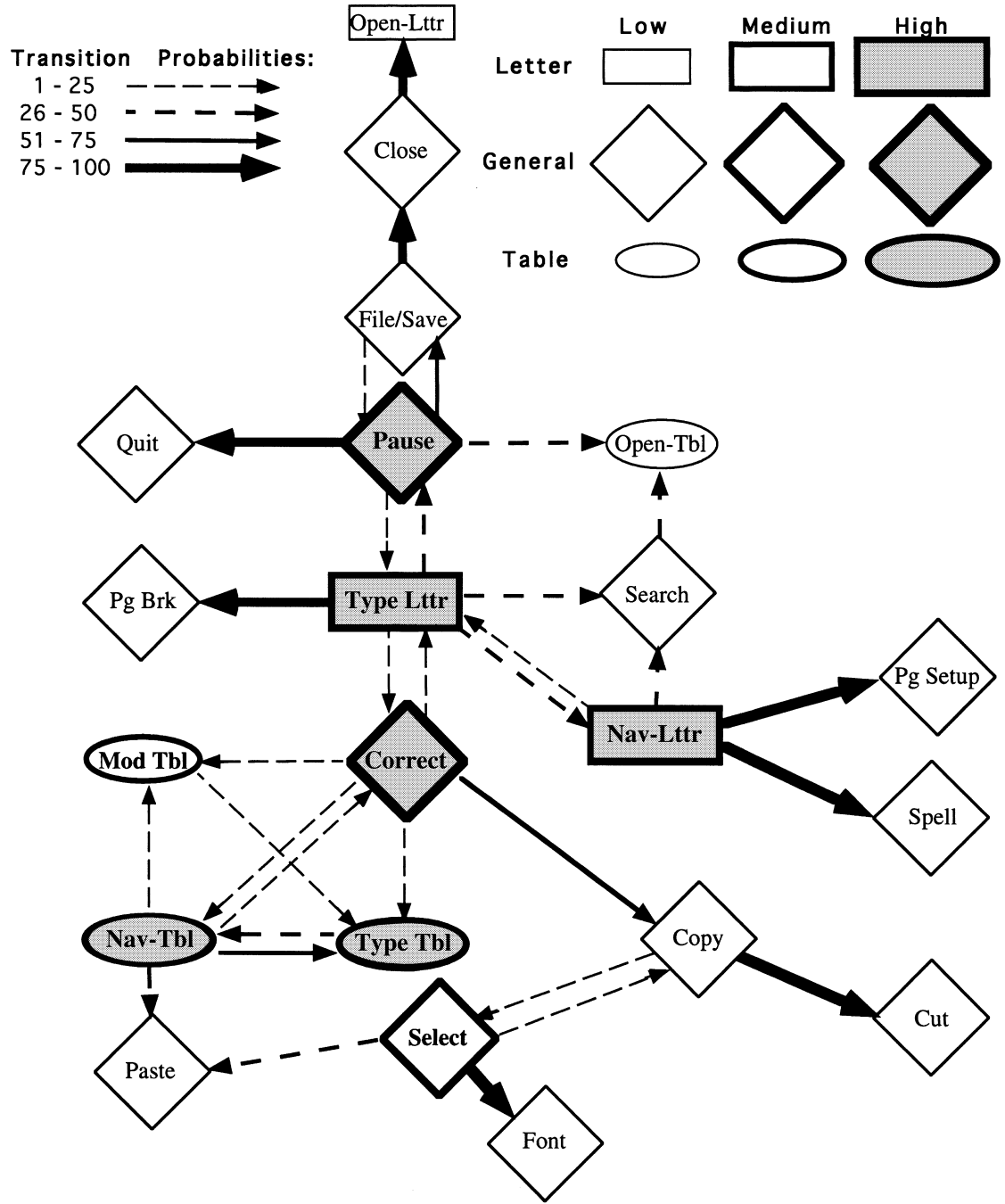

Fig. 3. Pathfinder network based on the conditional transition matrix for Windows-toWindows participant in Demonstration II. The shading of the nodes represents the type of behavior; the size of the node indicates the frequency of the behavior; and the thickness and dashedness of the lines linking nodes represents the level of conditional transition probability. 
The PRONET network for the Mac-to-Windows user (Fig. 4) consists of 25 nodes and 46 links (reduced from 25 functions which occurred a total of 172 times in 71 unique transitions between functions). The network has a degree of segregation between letter-related and table-related functions, with Typing, Navigating, and Modifying the Table grouped in a small subnetwork that also contains Correct and Select. Typing the letter is contained in a cycle with Correct, Cut, and Paste, and also links to Page Break, Page Setup and Spell Check. However, the other letter-related functions - Open Lttr and Nav Lttr are not linked directly to Type Lttr. For the Mac-to-Windows user, corrections were preceded by typing, either in the letter or the table, and, not surprisingly were often followed by more typing. In addition, corrections were followed by selecting text, cutting text, or modifying the table. This user engaged in a relatively large amount of searching, with Search as perhaps the central node of her network. One explanation for the pattern of behavior revealed by the network - widespread connections with Correct and Search, but appropriate use of functions - might be related to her previous experience with Word for the Macintosh, rather than with the Windows95 version used in this study. Both versions of Word allow the participant to perform most of the same functions by means of similar procedures. However, in Word for Windows, certain specific command names and the menu layout differ from Word for the Macintosh. Thus, this user appears to have had to engage in a substantial amount of searching, but typically located the appropriate function.

The behavior of the Mac-to-Windows user in this complex transfer task suggests she played an active role in transfer. Briefly, this user may have engaged in a substantial amount of searching because she knew what she wanted to do and had some idea of how to do it, but had to find a way to do it. In contrast, cognitive theories of transfer of training (cf. Polson, Muncher \& Englebeck, 1986; Singley \& Anderson, 1989) typically assume that: (1) task knowledge involves procedures consisting of rules that specify that when a condition is met, an action will be produced, and (2) transfer is based on the common procedures between the training and transfer tasks. To account for the behavior of the Mac-to-Windows participant, one might assume: (1) that she had organized knowledge (or mental models) about both the task and the system with which she had experience, and (2) that the task of creating a letter activated her task knowledge which led to the activation of her system knowledge (e.g. Gillan, 1997). She then may have used both types of knowledge to guide an active search for conditions that would permit the specific actions to accomplish her goals. The suggestion that a user knows the action to perform and searches for the condition that will permit that action to occur reverses the condition-action sequence found in common elements 


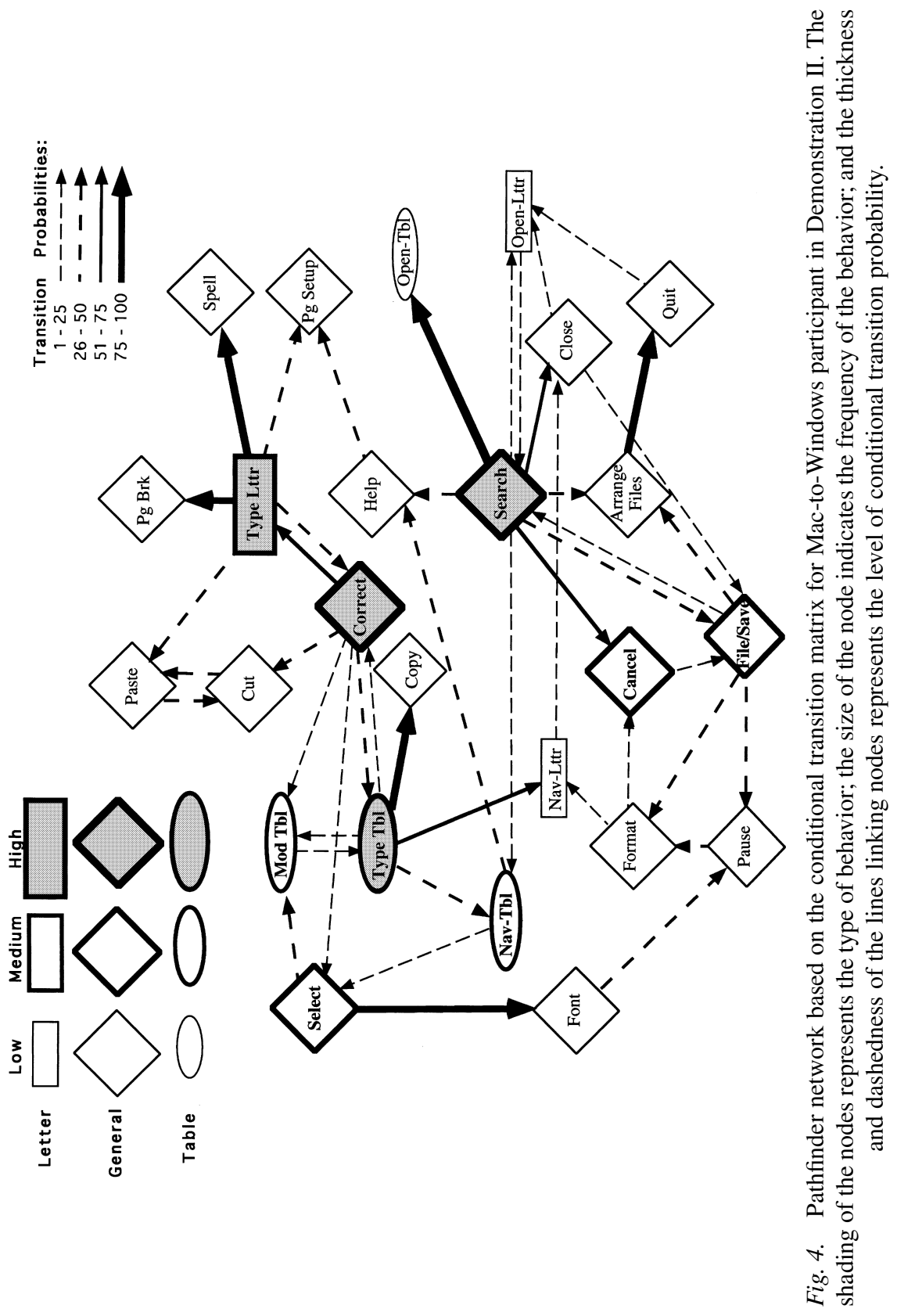


theories. This observation of the specific behaviors during transfer occurred because the PRONET method permitted a detailed examination of a user's behavior in a situation closely modeled after a real-world task; in contrast, most research on transfer only records performance measures like response time and errors.

\section{SELECTED EXAMPLES OF PRONET ANALYSIS OF COMPLEX BEHAVIORAL SEQUENCES}

In addition to the two demonstrations above, previously-published studies have applied the Pathfinder algorithm to event sequences as humans interact with technology. Like the above demonstrations, these studies support the thesis of this paper that PRONET networks provide a valid and useful representation of sequences of events in human-machine interaction research and design. They also illustrate an important aspect of the PRONET method - that it can be applied across a wide variety of situations and events, making it a valuable tool for investigations both in and out of the laboratory. Below we review two examples from these previously-published studies that used the PRONET approach in ways not shown in the demonstrations above. In the first study below, Cooke et al. (1996) created networks consisting of both environmental events (the information displayed by a computer) and human behaviors in response to those displays. In the second study, the researchers (Vortac, Edwards \& Manning, 1994) developed networks based on the behavior of teams as well as on individual's behavior.

Cooke et al. (1996) used PRONET to compare the behavior of student subjects as they acquired the ability to troubleshoot faults in the payload deployment and retrieval system (PDRS) of the Space Shuttle remote manipulator system (i.e. the robotic system in the shuttle) in a simulation. (The PDRS is used in deploying and stowing the manipulator arm in the Shuttle.) The simulation - a simplified version of an actual NASA mission control system - required that the user read information from a screen to identify a fault and to take action to correct the fault. The system cycled through the four stages of the PDRS - deploying a positioning mechanism, releasing latches, latching the latches, and stowing the positioning mechanism. The participants' task was to monitor the display, detect if a fault had occurred, identify the fault from a set of 13 possible faults, decide on the cause of the fault out of seven possible causes, and select a corrective action from 14 possible actions. At the end of each stage, the participant received feedback that specified if the troubleshooting decisions were correct and, if not, showed the correct alternative. 
The participants' behavioral sequences were encoded into discrete events with a screen event as an antecedent condition and each participant response as the consequent action(s). Thirty-seven different screen configurations served to denote separate conditions; actions corresponded to the 36 potential selections that the participant could make with a mouse click. Cooke et al. (1996) applied the Pathfinder algorithm to matrices of transitions between the 73 events (37 conditions and 36 actions) for three sessions as the participants learned to perform the troubleshooting task. In addition, based on an analysis of the task, the researchers determined the ideal sequence of behaviors and, based on it, developed an ideal PRONET network for this complex task. They reasoned that, if participants acquired new procedural knowledge during the 12 hours of training, then their networks should come to resemble the ideal network as correct links replace incorrect links.

Cooke et al.'s (1996) analysis of the Pathfinder networks showed systematic changes as predicted - more correct links, fewer incorrect links, and an increasing similarity between the participants' networks and the ideal network. In addition, the measure of similarity of the participants' networks with the ideal network (a measure called the $\mathrm{C}$ value) correlated highly with participants' performance during the complex task: $r$ 's of 0.86 with the percentage of correct fault identification, 0.58 with the percentage of correct diagnoses, and 0.58 with the percentage of correct actions taken. These high positive correlations indicate that as the participants' networks became more similar to the ideal, their performance improved.

Figures 5 and 6 show the Pathfinder networks from Sessions 1 and 3, respectively, redrawn from one of Cooke et al.'s (1996) participants. (The links that match those of the ideal network are shown by the solid lines, whereas the links that don't have a corresponding link in the ideal network are dashed. In addition, due to the visual complexity of the figures, we chose not to display the link weights.)

The networks are complex, but by focusing on specific aspects of the two networks, they can become interpretable. For example, look at the diagnosis of fault nodes (labeled with DI). By determining the nodes that link with the diagnosis nodes (that is, that have go from a condition node [C], an action node [A], or from an identification of fault node [ID] to the diagnosis node), we can see the final step before a fault diagnosis. In Session 1, the network shows 17 links into diagnosis nodes, with 10 matching the ideal network and 7 not matching a link from that network. In contrast, in Session 3, the network shows 13 links into diagnosis nodes, with 11 matches to the ideal network and 2 nonmatches. This observation suggests that, in this task, increased accuracy in 


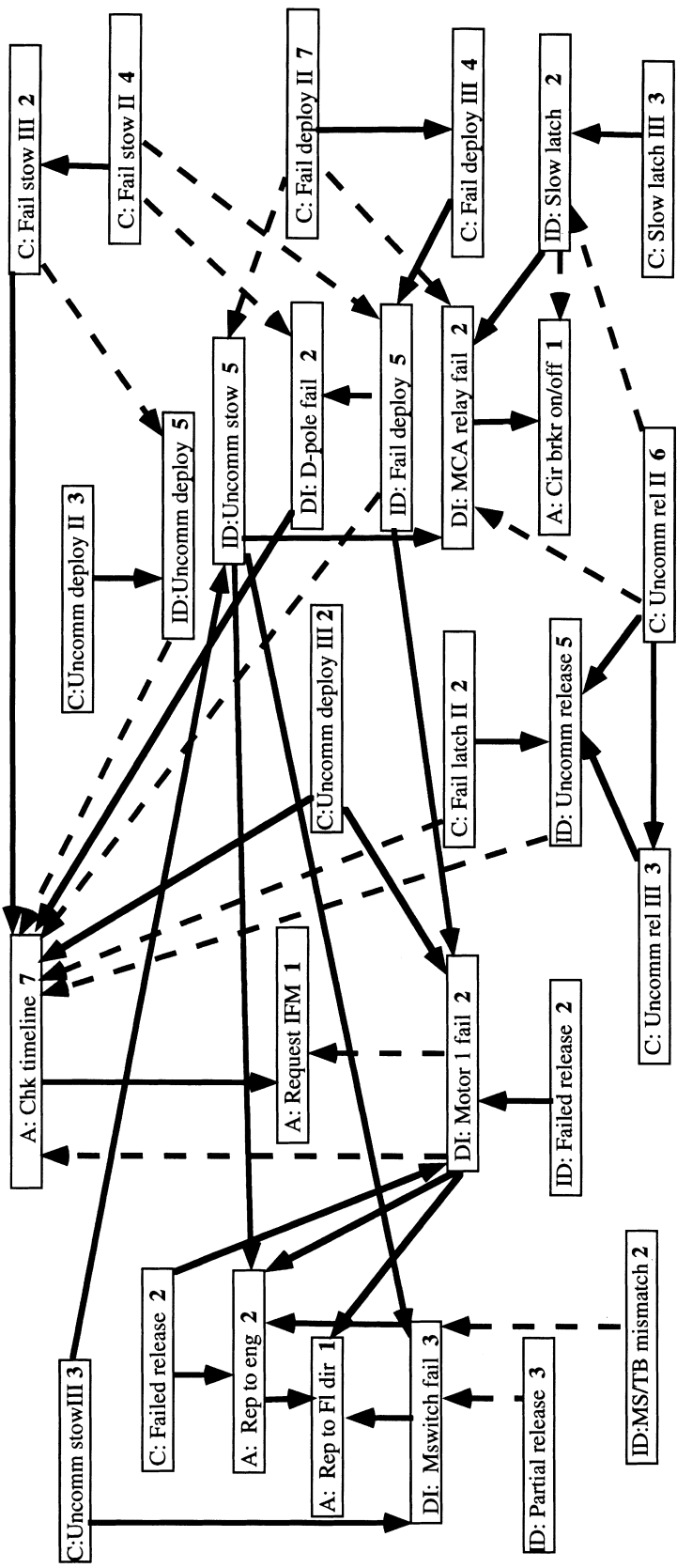

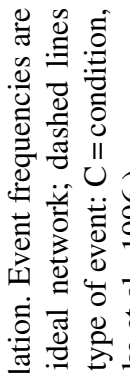

范 $\cong$

की

约

ง ลे

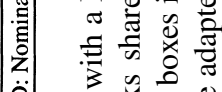

白

:

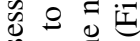

on

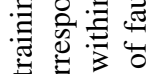

$\exists$ ठ क

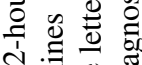

그욬

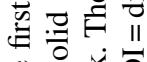

我菏

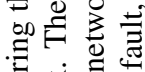

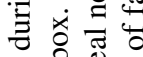

$\varangle$ :

ป Ð

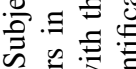

के

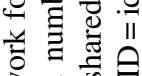

흥 ज

$\bar{t}$ 의

I $\Xi$ II

고원

๑ 귱

워 की

这运 


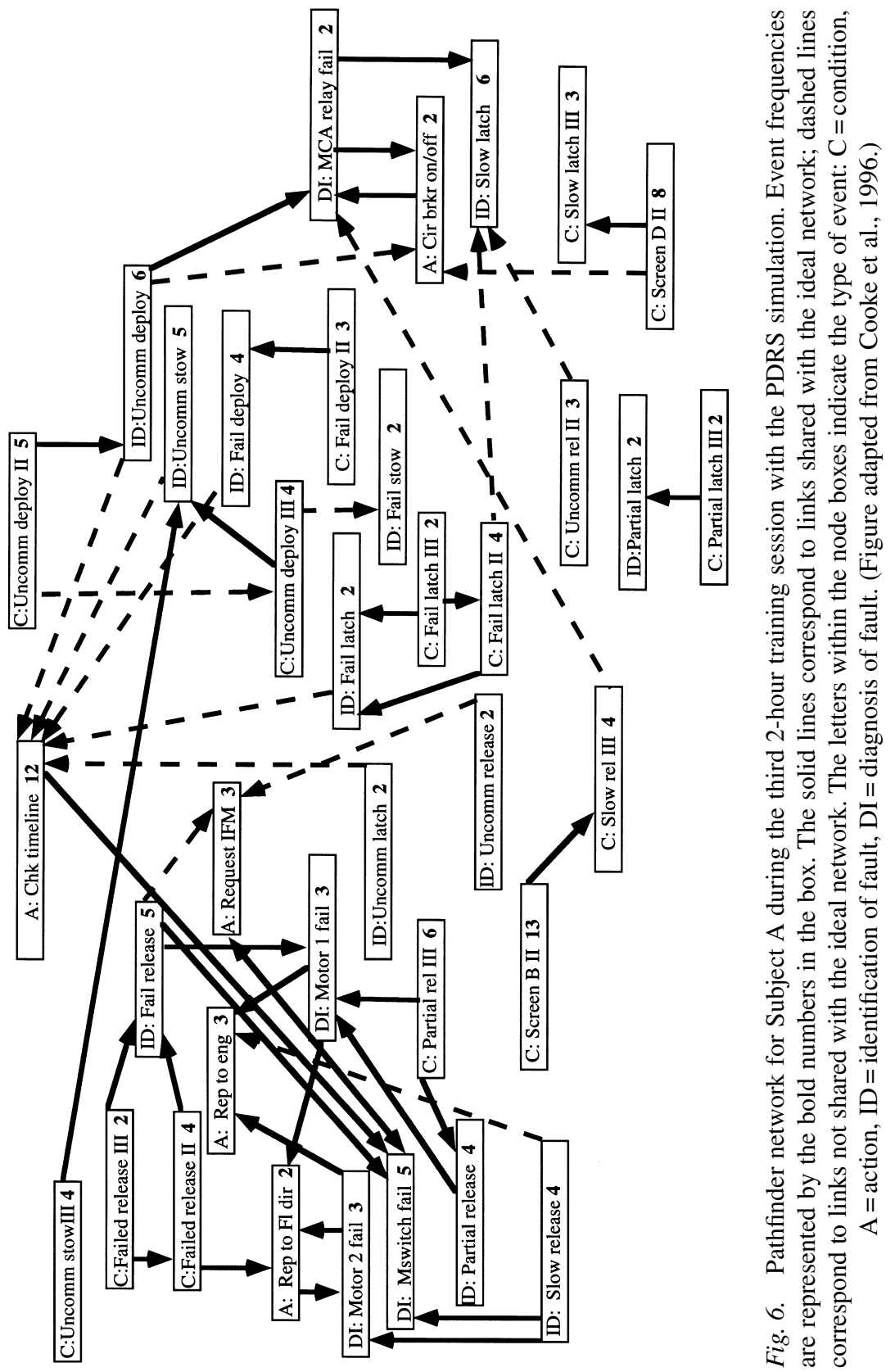


procedural knowledge is based largely on the loss of incorrect links or procedures (see also Anderson, 1983).

The second illustrative example comes from research on air traffic controllers by Vortac et al. (1994). One of the goals of the research was to use a Pathfinder network based on the frequencies of transitions between behaviors to study modules of cognitive processes and their relation to automation. Vortac et al. (1994) used the term module to mean a set of cognitive processes that frequently co-occur and have formed a single unit of cognition. Two important characteristics of a module are: (1) that the entire module has a single triggering event, rather than having separate triggers for each component cognitive process, and (2) effort is required if the module needs to be broken apart or fractionated. These modules resemble Anderson's (1983) construct of composed procedures - a set of procedures that becomes a unit due to repeated co-occurrence - and Hayes-Roth's (1977) construct of a cogit. One major concern of Vortac et al.'s (1994) was that implementing automation in a system should not disrupt any cognitive modules that the system operators had developed. That is, when introducing a new automated system, the underlying processes of the module or actions related to the module should not be distributed between operators or between the system and the operator.

In their research with air traffic controllers, Vortac et al. (1994) used Pathfinder networks to identify possible cognitive modules. They set the following criteria for this identification: (1) transitions between behaviors should occur frequently; (2) these transitions should be in close temporal proximity; (3) these transitions should occur in multiple situations; (4) the triggering event(s) should be both identifiable and reliable; (5) in a team task, one individual performs the actions related to the module, the actions are not distributed across the team. Thus, in order to apply these criteria, Vortac et al. (1994) recorded the behaviors of both individuals on two person teams, and then compared Pathfinder networks derived from individuals' behavior to networks derived from the team's behavior. In recording team behavior and deriving the Pathfinder network for this study, Vortac et al. (1994) kept track of the identity of the team member who produced the response. (However, researchers using Pathfinder to create a network of team behaviors could ignore the individual's identity. Given Vortac et al.'s goals in this study, being able to associate team actions with the actor was important.)

By comparing the individual and team networks, Vortac et al. (1994) found one set of behaviors that met the criteria for a cognitive module in a high fidelity simulation of controlling en route air traffic. The behaviors of verifying the information on a paper flight progress strip (FPS) or changing that information (what the researchers labeled as WRITE) were linked in the 
Pathfinder networks for both individuals and teams with behaviors for manipulating the FPS (e.g. moving it between posting boards in the ATC control area, sequencing a set of FPS's in a posting board, or removing the FPS) (what the researchers called MANIP). The co-occurrence of these two types of behavior was frequent, the two behaviors occurred close together in time, the transition between behaviors occurred across scenarios of varying complexity, from simple to complex. The sequence of WRITE - MANIP was triggered reliably by events related to transitions in flight control sectors, such as the initial contact with a sector. The sequence was also triggered, but somewhat less reliably, by a controller command to an aircraft. Finally, on a team, whichever individual engaged in a WRITE behavior, that same individual performed the subsequent MANIP behavior. The focus on FPS-relevant behaviors was important because the FPS system was being planned to be replaced by a computer-based and somewhat automated system. Vortac et al.'s (1994) results indicate that the new design of the FPS system should not distribute the WRITE and MANIP tasks between the human operator and the automated system.

\section{GENERAL DISCUSSION}

The demonstrations and examples described above show PRONET to be useful in summarizing, representing, and analyzing a diverse set of sequences of behavior - from a simulation of simple operant training of a bar press response (perhaps the most studied paradigm in all of experimental psychology), to using a word processor, to learning to diagnose faults in a Space Shuttle subsystem, to air traffic control. Aspects of the networks produced by the PRONET method were to some degree unsurprising. For example, the networks in the operant training sessions reflected the dramatic changes in behavior produced by changing the reinforcement contingencies. Likewise, the increasing similarity of networks to an ideal in the PDRS fault diagnosis learning task across the three sessions, and the closer similarity of experienced participants' networks to an ideal than an inexperienced participant in the transfer task all would be expected. So, a skeptic might say, "So what? PRONET just shows me what I already know". However, part of validation of a method involves demonstrating that the method can show you the expected results. Imagine, for example, if PRONET couldn't show the changes in behavior during operant training or showed greater similarity to an ideal sequence of behaviors for a novice than for an experienced word processor the method would rightly be judged as lacking. In addition, are there other methods that have been demonstrated to have the kind of range that PRONET 
has demonstrated in the three demonstrations above. A cumulative record could show the same increased bar pressing during operant training, but wouldn't show the effects seen in the more complex tasks; response time or accuracy measures would show the same performance effects during complex task training or transfer, but might not capture the full picture of operant training.

In the introduction, we criticized the standard measures used in cognitive psychology as being too simplistic to reflect the complexity of a sequence of behavior during a productive task in the work context. How did PRONET fare on that account? In the simple operant task, the PRONET networks revealed that several of the responses and most of the transitions between responses were not especially sensitive to the changes in reinforcement contingency. That is, the frequencies of drinking and licking did not decrease much as bar pressing became the dominant response, and the probability of a transition to sniffing remained high for most responses throughout the various sessions. This pattern of results would not have been noticeable with a cumulative record or response time measures. Similarly, the pattern of behavior of the Mac-toWindows user in the transfer demonstration that suggested that she was searching for the system-appropriate way to implement an action could be observed in the network. Measures like the overall task completion time or number of errors would have glossed over this observation. The importance of this observation is that it suggests that transfer involves an active learner, similar to the active nature of learners during acquisition (see Carroll, 1990).

\section{Benefits and Costs of PRONET}

An important criterion on which to evaluate any approach to data analysis focuses on the amount of effort required to perform the analyses in relation to the informational benefits that one gets from the analyses. The PRONET analysis reduced long and complicated streams of behavior to a network representation of those data that can be displayed on a single page. For example, the three participants analyzed in the transfer demonstration took between 30 and 45 minutes to complete the task and in doing so generated between 5300 and 10,500 lines of log file code recorded by the software. Any systematic analysis of this code, whether by the PRONET approach or any other system, would have to deal with this amount of data. Thus, although collection of data by automatically recording a user's behavior during a task either by videotaping or creating a computer log data file requires essentially no effort by researchers, any use of those data will be effortful. With PRONET, the path elimination feature inherent in the Pathfinder algorithm suggests that, as the complexity of the original data increases, the opportunity for path 
elimination also increases, thereby proportionally reducing the number of transitions in the network. Thus, the amount of data reduction makes it possible to collect large amounts of usability data in the field, while the methods of data reduction in PRONET may even make it advantageous to collect large amount of data.

An additional benefit of PRONET is its flexibility. The analyses in this paper looked only at first order transitions (lag of 1). That is, we counted the sequence of $\mathrm{A} \rightarrow \mathrm{B} \rightarrow \mathrm{C}$ as one increment in the frequency of co-occurrence matrix for $\mathrm{A} \rightarrow \mathrm{B}$, and one for $\mathrm{B} \rightarrow \mathrm{C}$, but none for $\mathrm{A} \rightarrow \mathrm{C}$. This latter case would be examined only if higher order transitions were included. In addition, we decided to delete from the networks any transitions that occurred less than $10 \%$ of the time. Although these decisions were not arbitrary, other approaches might be taken. The use of higher order transitions, examining multistep units of behavior (e.g. A-B-C instead of A-B and B-C), and various cutoffs for considering transitions as links could also be examined using PRONET.

If the effort to use PRONET were reduced, then the benefits of using PRONET - reduction in data and the increase in its interpretability and utility - would more strongly outweigh the cost in effort. The researchers spent most of their time on the projects described in the demonstrations encoding the behavioral data (e.g. chunking lines of log file code into categories that could be represented in a Pathfinder network as nodes). Although this process was effortful, the amount of time and effort is misleading because the encoding had to be done by hand and the coding scheme had to be enhanced concurrent with some of the encoding. The encoding process could be automated, for example, if the log file software recorded the data in a previously-developed coding scheme that was formatted by a PRONET tool for subsequent analysis by the Pathfinder algorithm. The PRONET tool would take the output after each session and use it to increment transition frequencies in an $N \times N$ matrix where $N$ represents the number of different events. Once the matrix was updated, the original $\log$ file data could be deleted, leaving behind only the frequency matrix.

\section{A Research and Development Agenda}

PRONET provides one method among several that have been developed in the behavioral and information sciences over the past 30 years for summarizing and visualizing event sequences. These methods include Markov modeling (e.g, Guzdial, 1993), lag sequential analysis (e.g. Sackett, 1978; Bakeman \& 
Gottman, 1997), Petri nets (Elwert, 1996; Palanque \& Bastide, 1996; Petri, 1980; and Rauterberg, 1993, 1995), and process discovery and validation (Cook \& Wolf, 1995, 1997). Each of these methods could be useful in helping cognitive researchers and user interface designers, as the demonstrations described above show PRONET to be (see also Gillan \& Cooke, 1998). Thus, one goal of future research should be to validate these methods for use in the study of cognitive systems in context. These methods should be examined using event sequence data from complex, productive tasks in the work context to determine: (1) the relation between the output of each method and performance measures, such as task completion time and task accuracy; (2) the degree to which each method represents the event sequence in a simplified and interpretable form, and (3) the ability for researchers to perform additional analyses on the output of each method.

A reasonable hypothesis is that the various methods - PRONET, Petri nets, Markov modeling, lag sequential analysis, and process discovery and validation - will be differentially useful as a function of the type of events, characteristics of the sequence, or the environmental conditions in which the events occurred. Thus, another goal for future research should be to identify the conditions under which each method would be most useful as a tool for representing and analyzing event sequences.

For PRONET to be more than simply a research instrument and to provide timely and useful information in typical task contexts, additional development will be required. Ideally, a system with the following characteristics will need to be developed: (1) the ability for the researcher, designer, or analyst to define the level of behavior to be recorded at the keystroke or functional level; (2) automatic recording of behaviors in sequence (much like a log data file); (3) organizing the sequence of behaviors in a transition matrix and a transition probability matrix, with the ability for the researcher, designer, or analyst to specify the lag size, and (4) automatic application of the Pathfinder algorithm, with the ability to set the $r$ and $q$ parameters. Such a system would allow the researcher, designer, or analyst to simply set the parameters, then after a session of user interaction, to examine the Pathfinder network. In the absence of such a system, much time and effort is required to prepare the data for analysis. However, we recognize that many important issues need to be addressed before this ideal system could be developed - primary among these is developing either: (1) an interface that permitted the researcher to specify functional categories and to map those categories onto keystroke level events, or (2) an intelligent system that could identify a set of keystroke level responses that would constitute a functional level category. 


\section{Guidelines for System Designers Using PRONET}

Perhaps the most important point for designers to keep in mind is that conducting and interpreting a Pathfinder analysis, whether of a sequence of behaviors or similarity ratings of a set of concepts, requires the acquisition of a substantial amount of skill. One cannot acquire that level of skill simply by reading a summary paper like this. Rather, that skill probably can be acquired best through practice with the method, reflection about the usefulness of the analysis, and feedback concerning the quality of the output of the analysis. Thus, we encourage potential users of PRONET to make use of the method initially to analyze tasks with a limited set of behaviors, like those in Demonstration I above, and tasks for which the analysis is not critical to the success or failure of a product. In addition, we caution first-time users of the method to expect that their early networks may be difficult to interpret; but with practice in the various elements of the PRONET approach, an analyst's skill will increase, leading to interpretable network representations of even complex behavioral sequences.

One key to applying PRONET successfully is in selecting the task. For example, complex, multifaceted work - such as work consisting of unrelated, concurrent tasks - would be less likely to produce useful networks than would more integrated tasks motivated by a single goal. Also, as we found with the operant conditioning and word processing demonstrations, the level of the task performer's skill will influence the network. Thus, a designer interested in the changes in behavior during skill acquisition should compare PRONET networks from users at various levels of experience. However, a designer interested in examining steady state behavior would be served best by collecting behavioral sequences from only more experienced users.

A second aspect to which PRONET users should attend is the level of analysis of the behavior. Our study of transfer of word processing knowledge that produced the data described in Demonstration II above provides a case in point. In analyzing the task, we parsed the behavioral sequences at two levels - a microscopic level (what Card et al., 1983, called "the keystroke level") and a more macroscopic level (Card et al.'s "functional level"). We undertook this dual analysis with the thought that the microscopic level analysis would be close to the level of the interface elements (e.g. the menu items and screen icons), whereas the macroscopic behaviors would be closer to the level at which the word processing participants conceived of the their work. Thus, to examine transfer of procedural knowledge, the functional level seemed to be a better choice. The key to deciding on the level at which to parse the behavior for PRONET is, first, to know how you plan to use the data. 
Third, PRONET users should decide on which lag(s) between behaviors will be most meaningful to analyze. Typically, most analysts will examine the lag 0 sequence (i.e. temporally adjacent responses). However, analysts in certain complex situations might find it enlightening to look at sequences of behaviors separated by one or more responses. For example, a task in which environmental events A and B (e.g. two different computer-sent messages) both produced the same immediate response (acknowledgment of receipt of the message), but produced reliably different second responses (e.g. initiating process $\mathrm{X}$ vs. terminating process $\mathrm{Y}$ ) would produce more meaningful networks with an analysis at a lag of 1 than at a lag of 0 . Given that the recording of the sequence of behaviors can be examined to produce a transition matrix at lag $=1$ or lag $=2$ as easily as lag $=0$, analysts may want to perform multiple analyses, each with a different lag value. Another option (done by in the research by Vortac et al., 1994, that we describe above) is to weight transitions of various lags differentially, then to combine the weighted transitions. Decreasing the weight as the temporal distance between behaviors increased would usually be the most useful approach.

A fourth important choice in applying PRONET is the selection of the $r$ and $q$ parameters in Pathfinder, which help to determine the density of the network. The simplest Pathfinder network will be produced with the settings of $r=$ infinity and $q=$ the number of behaviors -1 . However, as analysts acquire more ability, an approach to consider would be to create network of varying densities by varying the settings of $r$ and $q$.

Fifth, the interpretation of the network will be essential to the success of the PRONET analysis. PRONET provides a representation of a sequence of events, but the representation by itself has no meaning. The meaning that can be derived from the analysis comes from a clear understanding of your objectives in performing the analysis. In the absence of a concrete and well-formed goal, the likelihood of a PRONET network producing insight into user behavior is low. Given a clearly stated goal of the analysis, making use of interpretive heuristics (previously described in the above section, PRONET: Procedural Networks) should improve the quality of the interpretation. Briefly, those heuristics focused on extracting meaning from the elements of the network at various levels. Each link and the nodes that it connects represent a temporal relation between two events; the links with the highest weights would be those that had the highest probability of occurring. An environmental event leading to a behavioral event with a high link weight may reveal a user's procedural knowledge. Identify certain key events (especially those that might indicate an error or a problem) and find the events that consistently lead to or follow from those key events. Examine the network for events that have many connections 
because these will likely be central events (e.g. a choice point). The nodes and links may also be organized in structures, such as a chain of events (which would indicate a linear event sequence) or a subnetwork of tightly interconnected nodes (which might help you to identify a user-defined subtask).

In closing, we now have computer and videotaping technologies that permit us to collect massive amounts of observational data. Unfortunately, our development of data analytic tools has not kept pace with the data collection abilities. Ultimately, we need to be able to mine these data so that we can extract information about user's perceptual and cognitive processes. Progress in science and engineering depends on the development of good new methods. However, mass adoption of poor methods slows progress in science and engineering. The discipline of human factors in general, as well as the subdiscipline of human-computer interaction, have been willing to adopt new methods. Many of the new methods have at least been validated; positive examples of such methods include GOMS (e.g. Card, Moran \& Newell, 1983) and cognitive walkthroughs (e.g. Polson, Lewis, Rieman \& Wharton, 1992). Unfortunately, in other cases, the methods have been adopted without any assessment. We believe that researchers and practitioners in human factors should be as willing to evaluate the methods that they use as they are to evaluate user interfaces. In their classic paper, Gould and Lewis (1985) suggest that collecting user data is a key to successful usability engineering. We would amend that recommendation in two ways - that designers collect and analyze user data that is usable and interpretable for design.

\section{NOTE}

1. Pathfinder programs (i.e. KNOT) have been written in Pascal, C, Lisp, and APL. Various version of the program run on the IBM PC, Apple Macintosh, and SUN Microsystems computers. Information on obtaining programs is available from Interlink, Inc., P.O. Box 4086 UPB, Las Cruces, NM 88003-4086.

\section{ACKNOWLEDGMENTS}

The authors thank Microsoft for support of the research described in Demonstration II and for providing the software necessary for that study. Technical, logistic, conceptual, and design assistance was provided by Preston A. Kiekel, Shane Melton, Krisela Rivera, and Doug Reynolds. 


\section{REFERENCES}

Anderson, J. R. (1983). The architecture of cognition. Cambridge, MA: Harvard University Press.

Bakeman, R., \& Gottman, J. M. (1997). Observing interaction: An introduction to sequential analysis (2nd ed.). Cambridge: Cambridge University Press.

Bakeman, R., \& Quera, V. (1995). Analyzing interaction: Sequential analysis with SDIS and GSEQ. Cambridge: Cambridge University Press.

Card, S., Moran, T., \& Newell, A. (1983). The psychology of human-computer interaction. Hillsdale, NJ: L. Erlbaum.

Carroll, J. M. (1990). The Nurnburg funnel: Designing minimalist instruction for practical computer skill. Cambridge, MA: MIT Press.

Cook, J. E., \& Wolf, A. L. (1995). Automating process discovery through event-data analysis. Proceedings of the 17th Annual ICSE 'Conference on Software Engineering (pp. 73-82). New York: ACM.

Cook, J. E., \& Wolf, A. L. (1997). Controlling product evolution through process validation. Proceedings of the ICSE '97 Workshop on Process Modeling and Empirical Studies of Software Evolution (pp. 23-27). New York: ACM.

Cooke, N. J. (1992). Predicting judgment time from measures of psychological proximity. Journal of Experimental Psychology: Learning, Memory, and Cognition, 18, 640-653.

Cooke, N. J., Durso, F. T., \& Schvaneveldt, R. W. (1986). Recall and measures of memory organization. Journal of Experimental Psychology: Learning, Memory, and Cognition, 12, 538-549.

Cooke, N. J., \& Gillan, D. J. (1999). Representing human behavior in human-computer interaction. In: A. Kent \& J. G. Williams (Eds), Encyclopedia of Computer Science (pp. 283-308). New York: Marcel Dekker.

Cooke, N. J., Neville, K. J., \& Rowe, A. L. (1996). Procedural network representations of sequential data. Human-Computer Interaction, 11, 29-68.

Dunn-Rankin, P. (1983). Scaling methods. Academic Press: San Diego.

Elwert, T. (1996). Continuous and explicit dialogue modeling. Conference on Human Factors in Computing Systems CHI'96 Conference Companion . New York: ACM.

Gillan, D. J. (1997). The three psychologies of human-computer interaction. Proceedings of the Human Factors and Ergonomics Society 41st Annual Meeting (pp. 385-389). Santa Monica, CA: HFES.

Gillan, D. J., \& Cooke, N. M. (1998). Making usability data more usable. Proceedings of Human Factors and Ergonomics Society 42th Annual Meeting (pp. 300-304). Santa Monica, CA: HFES.

Gillan, D. J., \& Schvaneveldt, R. W. (1999). Applying cognitive psychology: Bridging the gulf between basic research and cognitive artifacts. In: F. T. Durso, R. Nickerson, R. Schvaneveldt, S. Dumais, M. Chi \& S. Lindsay (Eds), The Handbook of Applied Cognition (pp. 4-31). Chichester, England: Wiley.

Goldsmith, T. E., \& Davenport, D. M. (1990). Assessing structural similarity of graphs. In: R. W. Schvaneveldt (Ed.), Pathfinder Associative Networks: Studies in Knowledge Organization (pp. 179-195). Norwood, NJ: Ablex.

Gottman, J. M., \& Roy, A. K. (1990). Sequential analysis: A guide for behavioral researchers. Cambridge: Cambridge University Press. 
Graham, J., Alloway, T., \& Krames, L. (1994). Sniffy, the virtual rat: Simulated operant conditioning. Behavior Research Methods, Instruments, \& Computers, 26, 134-141.

Guzdial, M. (1993). Deriving software usage patterns from log files. GVU Center Technical Report No. 93-41. Atlanta, GA: Georgia Institute of Technology.

Hammontree, M., Weiler, P. and Hendrich, B. (1995). PDA-based observation logging. In: Human Factors in Computing Systems Chi '95 Conference Companion. New York: ACM.

Hayes-Roth, B. (1977). Evolution of cognitive structures and processes. Psychological Review, 84, 260-278.

Hutchins, E. (1996). Cognition in the wild. Cambridge, MA: MIT Press.

Johnson, S. C., (1967). Hierarchical clustering schemes. Psychometrika, 32, 241-254.

Kruskal, J. B., \& Wish, M. (1978). Multidimensional Scaling,. Beverly Hills, CA. Sage.

Mayer, R. E. (1999). Instructional technology. In: F. T. Durso, R. Nickerson, R. Schvaneveldt, S. Dumais, M. Chi \& S. Lindsay (Eds), The Handbook of Applied Cognition (pp. 551-569). Chichester, England: Wiley.

Millen, D. R., Schreifer, A., Lehder, D. Z., \& Dray, S. M. (1997). Mind maps and causal models: Using graphical representations of field research data. In: Human Factors in Computing Systems CHI'97 Conference Companion (pp. 265-266). New York: ACM.

Owen, R. N., Baecker, R. M., \& Harrison, B. (1994). Timelines, a tool for the gathering, coding, and analysis of temporal HCI usability data. In: Human Factors in Computing Systems Chi '94 Conference Companion. New York: ACM.

Palanque, P. and Bastide, R. (1996) Temporal aspects of usability: Time modeling in Petri nets for the design of interactive systems. SigCHI Bulletin, 28, 43-46.

Petri, C. A. (1980). Introduction to general net theory. In: W. Bauer (Ed.), Lecture Notes in Computer Science: Net Theory and Applications (pp. 1-21). Berlin: Springer-Verlag.

Pinker, S. (1997). How the mind works. New York: Norton.

Polson, P. G., Muncher, E., \& Englebeck, G. (1986). A test of a common elements theory of transfer. In: M. Mantei \& P. Obeton (Eds), Human Factors in Computing Systems CHI'86 (pp. 78-83). New York: ACM.

Rauterberg, M. (1993) AMME: An automatic mental model evaluation to analyze user behavior traced in a finite, discrete state space. Ergonomics, 36, 1369-1380.

Rauterberg, M. (1995). From novice to expert decision behavior: A qualitative modeling approach with Petri Nets. In: Y. Anzai, K. Ogawa \& H. Mori (Eds), Symbiosis of Human and Artifact: Human and Social Aspects of Human-Computer Interaction Vol. 2 (pp. 449-454). Amsterdam: Elsevier.

Rauterberg, M. (1996) The AMME Manual: A Petri net based analyzing and modeling tool kit for log files in human-computer interaction. Technical Report CC-5-96, Work and Organization Psychology Unit, Swiss Federal Institute of Technology, Zurich, Switzerland.

Reisig, W. (1992). A primer in Petri net design. Berlin: Springer-Verlag.

Sackett, G. P. (1978). Measurement in observational research. In: G. P. Sackett (Ed.), Observing Behavior (Vol. 2): Data collection and analysis methods (pp. 25-43). Baltimore: University Park Press.

Sanderson, P. M., \& Fisher, C. (1994). Exploratory sequential data analysis: Foundations. HumanComputer Interaction, 9, 251-317.

Schvaneveldt, R. W. (Ed.) (1990). Pathfinder associative networks: Studies in knowledge organization. Norwood, NJ: Ablex.

Schvaneveldt, R. W., Durso, F. T., \& Dearholt, D. W. (1989). Network structures in proximity data. In: G. H. Bower (Ed.), The Psychology of Learning and Motivation: Advances in Research and Theory (Vol. 24, pp. 249-284). New York: Academic Press. 
Singley, M. K., \& Anderson, J. R. (1989). The transfer of cognitive skill. Cambridge, MA: Harvard University Press.

Vortac, O. U., Edwards, M., \& Manning, C. (1994). Sequences of actions for individual and teams of air traffic controllers. Human-Computer Interaction, 9, 319-343. 